\title{
Differences in anosmic and normosmic group in bimodal odorant perception: a functional- MRI study*
}

\author{
Emilia Iannilli' ${ }^{1}$, Thomas Bitter ${ }^{2}$, Hilmar Gudziol' ${ }^{2}$, Hartmut P. Burmeister ${ }^{3}$, \\ Hans-Joachim Mentzel ${ }^{3}$, Anita P.S. Chopra ${ }^{4}$, Thomas Hummel ${ }^{1}$

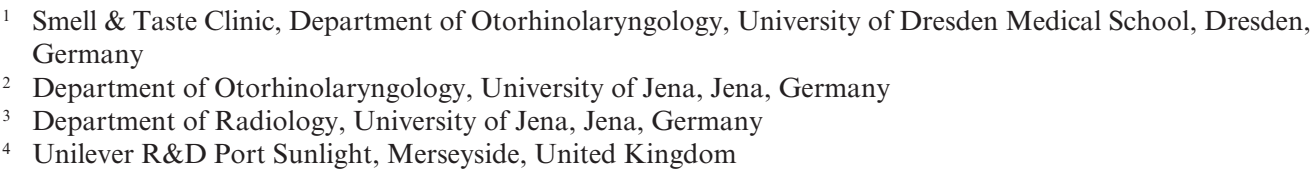

SUMMARY So-called bimodal odorants are able to stimulate the intranasal trigeminal system at relatively low concentrations. Using them as stimuli, the current study focused on the interaction between the olfactory and trigeminal systems at a cerebral level. In the experiment, menthol was used at two concentrations, low and high, and these were delivered to two groups of subjects, a healthy control group and an anosmic group who were unable to perceive smells. A computer-controlled olfactometer based on principles of air-dilution was used to deliver the stimuli, while the brain functions were assessed by a functional magnetic resonance imaging ( $f M R I)$ technique. SPM5 was used for data analysis. The results showed that normosmic subjects exhibited activation in the anterior cingulate cortex $(A C C)$ and posterior cingulate cortex (PCC), prefrontal cortex $(P F C)$, and cerebellum. Whilst anosmic subjects activated the same area inside the anterior cingulate; moreover a cluster of activation was found in the left parahippocampal gyrus. In controls, an effect of stimulus intensity was localized between the anterior cingulated, the medial frontal gyrus and the cerebellum; such areas could not be found in anosmic subjects. These results suggest that the olfactory system modifies trigeminally mediated information causing an evident effect in the differentiation between stimulus intensities.

Key words: olfaction, smell, irritation, trigeminal, anosmia

\section{INTRODUCTION}

Most odorous compounds stimulate both olfactory and intranasal trigeminal receptors ${ }^{(1,2)}$, at least when presented at higher concentrations. Menthol is a chemical compound, which stimulates both trigeminal free nerve endings in the nasal cavity and the olfactory receptor neurons in the olfactory mucosa; at very low concentrations this is an example of a bimodal odorant. On the other hand, the trigeminal and the olfactory system interact, mutually suppressing and enhancing each other ${ }^{(3-5)}$. The interaction can take place at the cerebral level, at the level of the olfactory bulb, or at the level of the nasal epithelium ${ }^{(6,7)}$. It is still not entirely clear which brain areas relate to the processing of information specifically within each system or which areas would relate to interactions between the two systems. To further investigate the interaction between the two systems, a study based on functional magnetic resonance imaging (fMRI) was set up. Chemical stimuli were presented at two concentrations to two groups of subjects, a healthy control group and an anosmic group. Anosmic patients have no functioning olfactory system, but they can detect a large range of odorants presented to the nose, presumably via the somatosensory input through the trigeminal nerve ${ }^{(2)}$.

Considering that there was practically no central olfactory processing in the anosmic group, the hypothesis was that the comparison between the two samples could highlight or hide specific brain areas directly involved with one of the chemical perceptive systems. Moreover, to investigate an overlap or eventually a modulation between the two systems, the bimodal odorant was delivered at two different concentrations. 


\section{MATERIALS AND METHODS}

\section{Subjects}

The two study groups consisted of 17 anosmic subjects (11 women, mean age \pm SE is $48 \pm 4$ years) and 17 normosmic subjects for the control group (11 women, mean age \pm SE is 41 \pm 4 years). For the pathological group, 5 subjects had a postinfectious loss of sense of smell, 5 presented with anosmia after a closed head trauma, and 7 patients were diagnosed with idiopathic anosmia. Olfactory function of all subjects was evaluated by the "Sniffin' Sticks" test ${ }^{(8)}$. Furthermore, a detailed medical history was performed and subjects received an endoscopic examination of the nasal cavity.

\section{Stimuli and delivering methods}

The volatile chemical stimulus menthol was presented in two concentrations, menthol-low (M-low) $50 \% \mathrm{v} / \mathrm{v}$ and mentholhigh (M-high) 66\% v/v. A MRI-compliant computer-controlled air-dilution olfactometer $(80 \%$ relative humidity; total flow $7 \mathrm{~L} / \mathrm{min}$; $\left.36^{\circ} \mathrm{C}\right)(\mathrm{OM} 6 \mathrm{~B}$ : Burghart instruments, Wedel, Germany) was used to present the odour to the subjects' right nostril. It has to be noted that the stimuli were not perceived as painful unlike $\mathrm{CO} 2$-stimuli, which have been used in previous studies investigating interactions between the olfactory and the trigeminal systems (e.g., $\left.{ }^{(9)}\right)$.

\section{fMRI protocol and device}

The blood oxygenation level dependent (BOLD) signal for the fMRI images was acquired by means of 3T MRI-scanner (Siemens Magnetom Trio Tim System 3T). The functional images were collected in 120 volumes/session using a 36 axialslice matrix 2D SE/EP sequence (Matrix: 128 x 128; TR: 2 sec; TE: $30 \mathrm{~ms}$; FA: $90^{\circ}$; Voxel size: $1.72 \times 1.72 \times 2 \mathrm{~mm}$ ). The fMRI protocol was built in block-design (Figure 1). In the on-block one of the two stimulus conditions was sent with the following parameters: stimulus duration $250 \mathrm{~ms}$, randomized inter-stimulus interval between $1 \mathrm{~s}$ and $2.5 \mathrm{~s}$. In the off block, the subject perceived only humidified airflow. The total experiment was composed of two sessions with 6-block on-off per session. The two stimulus conditions, M-low and M-high, were randomized across the blocks and every condition was repeated 6 times.

\section{Data analysis}

The fMRI data analysis was performed using the SPM5 software package (Statistical Parametric Mapping; Wellcome Department of Cognitive Neurology, London, UK) implemented in Matlab 6.5 R.13 (Math Works Inc., Natick, MA, USA). After the spatial pre-processing (registering, realignment, co-registration between functional and structural images, normalization in a stereotaxic space, and smoothing) ${ }^{(10)}$. A first level statistical analysis was carried out with the canonical hemodynamic function available in SPM5. The group analysis was carried out by a random-effects analysis ${ }^{(11)}$ and was modelled using an ANOVA 2 x 2, 2 factors (stimulus, group) x two level each factor. The statistical threshold was set at $\mathrm{p}_{\text {FWE-corr }}<$ 0.05 , and cluster lever of $\mathrm{k}=3$ voxels.

\section{RESULTS \\ Rating}

After each session, subjects were asked to evaluate the stimuli in terms of intensity in a score range between ' 0 ' $=$ 'not perceived' and ' 10 ' = 'extremely intense.'

The statistical analysis revealed that when menthol was presented the anosmic subjects perceived it and rated it (M-low: mean $\pm \mathrm{SE}$ is $3.0 \pm 0.5$; M-high: mean $\pm \mathrm{SE}$ is $3.0 \pm 0.7$ ) but they were not able to distinguish between the high and the low concentration [T-test: $\mathrm{p}=0.66, \mathrm{t}=0.44, \mathrm{df}=30$ ]. On the other hand, normosmic subjects perceived the two concentrations as two distinct intensities (M-low: mean $\pm \mathrm{SE}$ is $2.0 \pm 0.4$; M-high: mean $\pm \mathrm{SE}$ is $4.0 \pm 0.4$ ) [T-test: $\mathrm{p}=0.0097, \mathrm{t}=2.76$, df $=30]$ (Figure 2).

\section{Functional images analysis}

Focusing on the control group (normosmic $=\mathrm{N}$-group), the effect of menthol was highlighted inside the anterior (ACC) and posterior cingulate (PCC) cortex, inside the prefrontal cortex (PFC) and the cerebellum (Table 1). The anosmic patients activated the same area inside the ACC/medial frontal gyrus (Table 2) with larger clusters compared with the N-group. Moreover, a cluster of activation was found inside the left parahippocampal gyrus. No primary olfactory cortex was highlighted presumably because of the large susceptibility artefact, at magnetic field of $3 \mathrm{~T}$, in brain areas close to airfilled structures, like the orbitofrontal cortex where the major olfactory-eloquent areas are located.

The difference in activations between the two groups (Table 3 and Figure 3) was evaluated at the statistical level of $\mathrm{p}_{\text {FWE-corr }}$ $=0.05$. Then in the direction N-group (minus) A-group, the statistical parametrical map showed one cluster of activation located inside the cerebellum. On the opposite direction the contrast A-group (minus) $\mathrm{N}$-group revealed activations in the frontal lobe, DLPFC (BA9) and ACC (BA 32).

Finally, the effect of concentration was evident for the $\mathrm{N}$-group in a cluster ( 4 voxels, $\mathrm{T}=4.41, \mathrm{p}_{\mathrm{FWE}-\mathrm{corr}}=0.034, \mathrm{df}=$ 58 ) localized in the right anterior lobe of cerebellum (max: $4-$ $48-2$ ) activated more strongly by the high concentration of the stimulus compared with the low concentration. Vice versa in the $\mathrm{N}$-group the contrast low vs. high concentration activated a large cluster localized between the ACC (Brodmann area: BA32) and the medial frontal gyrus (BA10) (max: 050 12; $\mathrm{T}=$ $4.41, \mathrm{p}_{\text {FWE-corr }}=0.038, \mathrm{df}=58$ ) (Figure 4). On the other hand, the A-group did not show any activated voxels contrasting low vs. high concentration and vice versa.

\section{DISCUSSION}

This functional imaging study focused on two different populations (N-group and A-group) to investigate effects related to a complete loss of the trigeminal or olfactory system. In our study, we were interested in olfactory loss. Two different concentrations of a bimodal odorant were applied. It was hypothesized that such a design could highlight differences at 


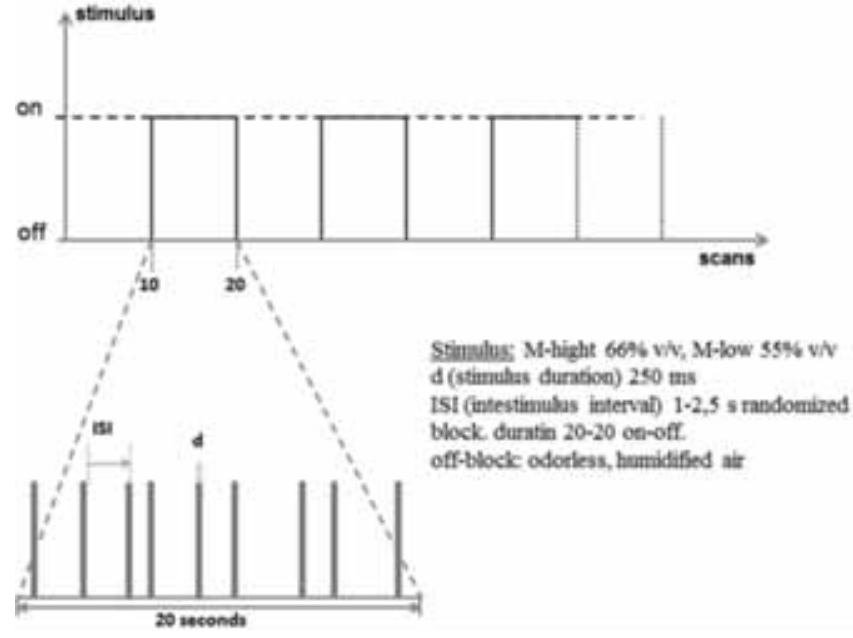

Figure 1. Details of Experimental fMRI-paradigm.

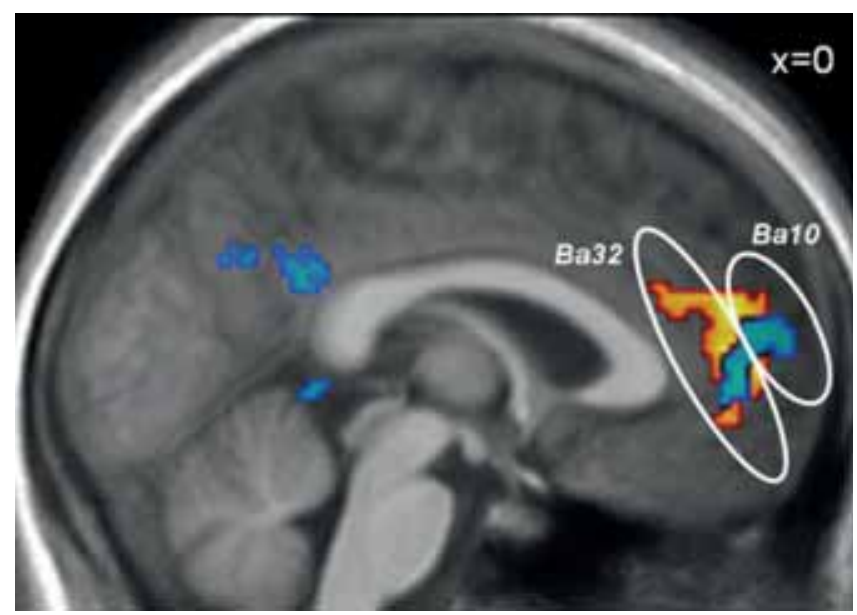

Figure 3. Activations following the Menthol stimulus for N-group (in blue) and A-group (in red). The A-group shows a bigger activation inside an area between the anterior cingulate the frontopolar cortex rather than the $\mathrm{N}$-group that covers the same areas but with a little extension and more toward the frontopolar portion. (pFWE-corr < $0.05, \mathrm{k}=3$ voxel,- MNI coordinates $)$.

a central level between the olfactory and the trigeminal system, and further elicits the brain areas involved in the intensity evaluation of the stimulus.

The analysis of intensity ratings of the two levels of stimuli revealed that the A-group was unable to distinguish between the low and the high concentration of the stimuli even though they claimed to be able to perceive and evaluate it. In contrast, the N-group perceived the stimulus at high concentration more intense than the low concentration. This can be interpreted such that A-group subjects became less sensitive and less discriminative towards trigeminal stimuli as had been shown earlier $^{(4,12,13)}$.

The fMRI analysis showed in both groups a wide-spread acti-

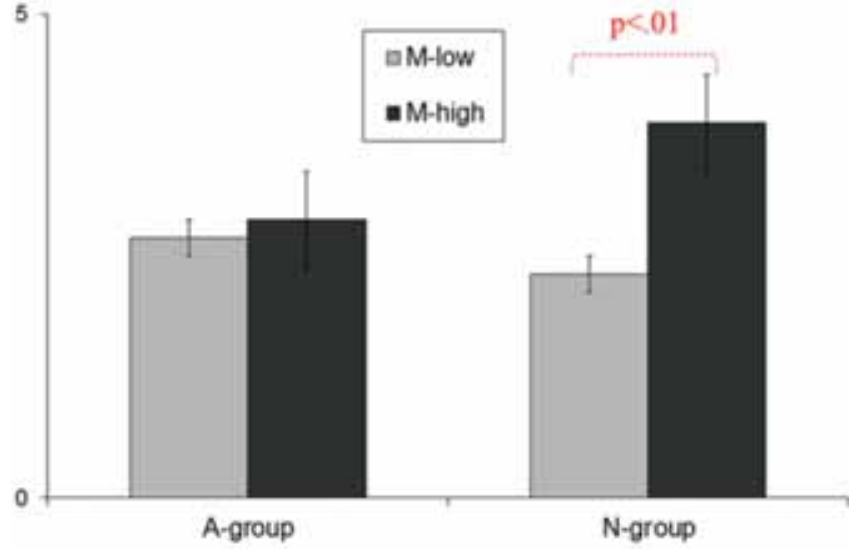

Figure 2. Intensity ratings performed by the two groups, Anosmic subjects and Controls. The A-group was not able to distinguish between the two intensities [T-test: $\mathrm{p}=0.663, \mathrm{t}=0.441, \mathrm{df}=30$ ], whereas the controls did [T-test: $\mathrm{p}=0.00973, \mathrm{t}=2,76, \mathrm{df}=30$ ].

vation in the ACC (BA32) and prePFC (BA10). This cluster was bigger in the A-group compared to the $\mathrm{N}$-group, a large overlapping area could be observed (Figure 3). Interestingly, this area was also found by Savic et al., ${ }^{(14)}$ when healthy subjects were stimulated with acetone or butanol, which are also bimodal odorants, but not when the stimulus was vanillin, a selective olfactory stimulant ${ }^{(15)}$. Furthermore, the ACC is well known to be involved in the processing of painful and noxious stimuli ${ }^{(16-19)}$. Based on these previous studies and on our results, considering that this area was present in both groups, we concluded that the involvement of the ACC is connected with the processing of the cooling-irritative trigeminal component of menthol. Although the A-group seems to react with a big cluster of activation in this area the comparisons between the two intensities did not produce any response in these areas, suggesting again a loss of discrimination and sensibility towards the chemosensory stimulus, not only to the smell component but also to the trigeminal one.

Additionally, the A-group showed activation in the parahippocampal gyrus (BA 30), an area which is correlated with olfactory memory, semantic memory and recollection vs. familiarity ${ }^{(20)}$ as well as with odour familiarity ${ }^{(21)}$. In our study, only subjects were included in the A-group who reported that they were subjectively normosmic before they became anosmic. Furthermore, in all subjects olfactory loss occurred during adulthood. Thus, it can be expected that all of these patients were familiar with the olfactory portion of the sensation elicited by menthol. Considering this, we hypothesize that the activation in the parahippocampalgyrus in our task was associated with the subject's attempt to 'recall' the formerly known menthol smell sensation based on the perception of the trigeminal component of menthol.

A direct statistical parametrical map comparison between two groups showed a positive effect for the N-group at the cerebellum level, which was absent for the A-group. The involve 
a) Menthol-low vs. high
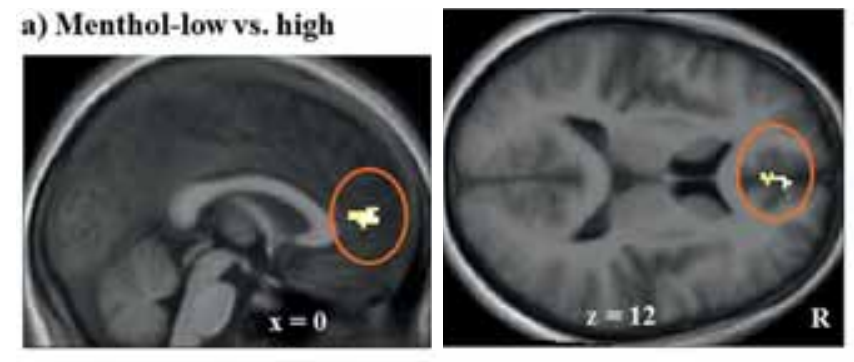

b) Menthol-high vs.low
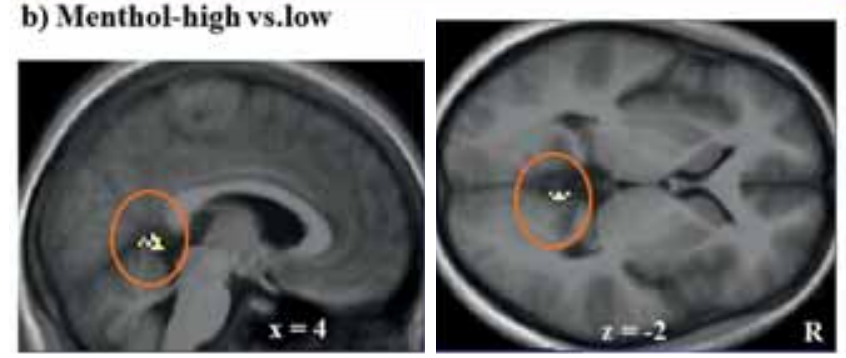

Figure 4. Brain activationscorrelated to the effect of the intensity of the stimulus for the N-group. (a) Contrasting menthol at high concentration vs. menthol at low concentration it was highlighted a cluster with a maximum in the voxel $\left(\begin{array}{l}0 \\ 0\end{array} 0\right.$ 12) (pFWE-corr $\left.=0.038\right)$ and it cover the regions between the BA32 (anterior cingulate cortex) and the BA10 (Med. Frontal G.). (b) Vice versa contrasting menthol low vs. menthol high it was emphasized a cluster with maximum $(4-48-2)$ (pFWEcorr $=0.038, \mathrm{~T}=4, \mathrm{df}=58$ ) inside the cerebellum in the anterior lobe. $\mathrm{R}=$ right hemisphere. Coordinate in MNI space.

ment of the cerebellum in olfactory function is still a matter of interest. There are evident results indicating an important role for the cerebellum in smelling/sniffing (?); for example Mainland at al., (22) demonstrated in their work that cerebellar lesions impacted on olfactory and olfactomotor performance. Applegate and Luis ${ }^{(23)}$ found a mild olfactory dysfunction in a cerebellar disorder as well as Connelly et al., (24) on a group affected by ataxias. Our results confirm this observation stressing the hypothesis that the cerebellum plays an important role in chemosensory processing.

Another difference between the two groups (that is A-group (minus) $\mathrm{N}$-group) is presented by a direct measure of the activated voxels

surplus for the A-group, inside the area covering the ACC and BA9, and common to the two groups. As we have already discussed, this area seems to be related to the irritating component of the stimulus; the increased activation for the A-group suggests a local stronger response to the trigeminal stimulus that nevertheless does not correspond to a stronger sensation of it, as it is shown in the psychophysical results. On the other hand, electrophysiological studies have found a reduced event related potential (ERP) response for acquired anosmia (AA) compare to normosmic subject when stimulated with pure trigeminal stimulus such as $\mathrm{CO}_{2}{ }^{(25)}$. There are two main reasons for this discrepancy. First, in our experiment we used a bimodal odor- ant that could be processed differently from a pure trigeminal stimulus because of the two components. This could imply that the processing of the olfactory component for the N-group works slightly to the detriment of the trigeminal one in this brain area. Second, we have to consider that a larger cluster in fMRI does not always correspond to a higher amplitude in ERPs. This is because the pyramidal neurons, representing the electrical source, may be folded in the cerebral cortex following the folds of the brain and this can lead to delete the out coming electrical signal and then reduce the total amplitude. That means that if the brain area that is working is bigger, as observed in the A-group, it may involve more folds of the brain, and therefore a lower ERP signal.

The results of the functional images after the effect of the stimulus intensity showed for the A-group no supra-threshold voxel surviving, which confirms the psychophysical results. For the $\mathrm{N}$-group, the contrast low-vs-high concentration produced an evident activation inside an area spread between the ACC (BA32) and the medial frontal gyrus (BA10). This is in agreement with Royet et al., ${ }^{(26)}$ who correlated this area to the evaluation of odour intensity and hedonic impressions. Moreover, Rolls et al., (27) found this area specifically activated in reaching a decision about the physical properties of an odorous stimulus, such as intensity. In addition, the reverse contrast (high vs low concentration) revealed a cluster of activation inside the right anterior lobe of cerebellum, which is typically activated in nociceptive but as already discussed, also in olfactory stimuli ${ }^{(4,16,28,29)}$. Moreover, Bensafi et al., ${ }^{(17)}$ found in a recent study activation in the cerebellum in connection with the evaluation of the stimulus olfactory intensity. Thus, there is more evidence of the important role of the cerebellum in olfactory processing.

In conclusion, in the present study we focused on the interaction between the two major channels of chemosensory perception at the level of the central nervous system. Anosmic and Normosmic groups were compared under similar bimodal odorant stimulation. As main results, we confirm the involvement of the cerebellum in olfactory processing and the close cooperation between the trigeminal and olfactory systems at a cerebral level, resulting in more efficient information processing related to differentiation between stimulus intensities.

\section{ACKNOWLEDGEMENTS}

This work was supported by a grant from the DFG (SPP 1392: HU 441/10-1) to TH.

\section{AUTHORSHIP CONTRIBUTION}

Study concepts: EI, TH, TB, HG; Study design: EI, TH; Literature research: EI, TH; Data analysis/interpretation: EI, TH; Statistical analysis: EI; Manuscript preparation: EI, TH; Manuscript editing: EI; Subject enrollment: TB, HG; Data acquisition: HPB, HJM; Chemical stimuli preparation: AC; Manuscript revision/review and final version approval: all authors. 
Table 1. Statistical significant activated voxels following the contrast Menthol for the N-Group.

\begin{tabular}{|c|c|c|c|c|c|c|}
\hline \multirow[b]{2}{*}{ \# of cluster } & \multirow[b]{2}{*}{$\mathrm{p}$ (FWE-cor) } & \multirow[b]{2}{*}{$\mathrm{T}$} & \multirow{2}{*}{$\frac{\mathrm{MNI}}{\mathrm{x}, \mathrm{y}, \mathrm{z}(\mathrm{mm})}$} & \multirow{2}{*}{\multicolumn{3}{|c|}{ Brain area }} \\
\hline & & & & & & \\
\hline 22 & 0.015 & 4.67 & 2460 & limbiclobe & anteriorcingulate & BA 32 \\
\hline 24 & 0.024 & 4.52 & -25612 & frontallobe & $\begin{array}{c}\text { medialfrontal } \\
\text { gyrus }\end{array}$ & BA 10 \\
\hline 44 & 0.033 & 4.43 & $2-48-2$ & cerebellum & anteriorlobe & culmen \\
\hline 3 & 0.034 & 4.41 & $0-6628$ & occipitallobe & precuneus & BA 31 \\
\hline 10 & 0.039 & 4.38 & $0-48 \quad 24$ & limbiclobe & posteriorcingulate & BA 23 \\
\hline
\end{tabular}

$(\mathrm{df}=58$, with a pFWE-corr $<0.05$, cluster level $\mathrm{k}=3$ voxel, $\mathrm{BA}=$ Brodmann area $) *$

Table 2. Statistical significant activated voxels following the contrast Menthol for the A-Group.

\begin{tabular}{|c|c|c|c|c|c|c|}
\hline \multirow[b]{2}{*}{ \# of vocels } & \multirow[b]{2}{*}{$\mathrm{p}$ (FWE-cor) } & \multirow[b]{2}{*}{$\mathrm{T}$} & MNI & & & \\
\hline & & & $\mathrm{x}, \mathrm{y}, \mathrm{z}(\mathrm{mm})$ & \multicolumn{3}{|c|}{ Brain area } \\
\hline \multirow[t]{3}{*}{126} & 0.004 & 5.10 & 2466 & limbiclobe & anteriorcingulate & BA 32 \\
\hline & 0.007 & 4.74 & 34618 & frontallobe & $\begin{array}{c}\text { medialfrontal } \\
\text { gyrus }\end{array}$ & BA 9 \\
\hline & 0.038 & 4.38 & $-4 \quad 52 \quad 2$ & limbiclobe & anteriorcingulate & BA10 \\
\hline 7 & 0.049 & 4.29 & $\begin{array}{lll}-9 & -39 & 2\end{array}$ & Limbiclobe & $\begin{array}{c}\text { parahippocampal } \\
\text { gyrus }\end{array}$ & \\
\hline
\end{tabular}

$\left(\mathrm{df}=58\right.$, with a $\mathrm{p}_{\mathrm{FWR}-\text { corr }}<0.05$, cluster level $\mathrm{k}=3$ voxel, $\mathrm{BA}=$ Brodmann area $)$

Table 3. Statistical significant activated voxel in the difference between N-Group and A-Group.

\begin{tabular}{|c|c|c|c|c|c|c|}
\hline & & & MNI & & & \\
\hline \# of voxels & $\mathrm{p}$ (FWE-cor) & $\mathrm{T}$ & $\mathrm{x}, \mathrm{y}, \mathrm{z}(\mathrm{mm})$ & \multicolumn{3}{|c|}{ Brain area } \\
\hline \multicolumn{7}{|c|}{ N-group (minus) } \\
\hline \multicolumn{7}{|c|}{ A-grup } \\
\hline 13 & 0.045 & 4.31 & $\begin{array}{lll}2 & -48 & -2\end{array}$ & cerebellum & anterior lobe & \\
\hline \multicolumn{7}{|c|}{ A-gruop (minus) } \\
\hline \multirow[t]{2}{*}{9} & 0.018 & 4.63 & $346 \quad 20$ & frontallobe & $\begin{array}{l}\text { medial frontal } \\
\text { gyrus }\end{array}$ & BA 9 \\
\hline & 0.043 & 4.31 & $\begin{array}{lll}0 & 54 \quad 20\end{array}$ & & $\begin{array}{c}\text { medial frontal } \\
\text { gyrus }\end{array}$ & \\
\hline 3 & 0.023 & 4.52 & $244 \quad 12$ & limbic lobe & anterior cingulate & BA 32 \\
\hline
\end{tabular}

The statistical level was fixed at $\mathrm{p}_{\mathrm{FWE} \text {-corr }}=0.05$, cluster level $\mathrm{k}=3, \mathrm{BA}=$ Brodmann area.

\section{CONFLICT OF INTEREST}

The authors state no conflict of interest.

\section{REFERENCES}

1. Elsberg CA, Levy I, Brewer ED. The sense of smell VI. The trigeminal effects of odorous substances. 1935.

2. Doty RL, Brugger WE, Jurs PC, Orndorff MA, Snyder PJ, Lowry LD. Intranasal trigeminal stimulation from odorous volatiles: psychometric responses from anosmic and normal humans. Physiol Behav. 1978; 20: 175-85.

3. Livermore A, Hummel T. The influence of training on chemosensory event-related potentials and interactions between the olfactory and trigeminal systems. Chem Senses. 2004; 29: 41-51.

4. Iannilli E, Gerber J, Frasnelli J, Hummel T. Intranasal trigeminal function in subjects with and without an intact sense of smell. Brain Res. 2007; 1139: 235-244.

5. Cain WS, Murphy CL. Interaction between chemoreceptive modalities of odour and irritation. Nature. 1980; 284: 255-257.
6. Schaefer ML, Bottger B, Silver WL, Finger TE. Trigeminal collaterals in the nasal epithelium and olfactory bulb: a potential route for direct modulation of olfactory information by trigeminal stimuli. J Comp Neurol. 2002; 444: 221-226.

7. Hummel T, Doty RL, Yousem DM. Functional MRI of intranasal chemosensory trigeminal activation. Chem Senses. 2005; 30 Suppl 1: i205-i206.

8. Hummel T, Sekinger B, Wolf SR, Pauli E, Kobal G. 'Sniffin' sticks': olfactory performance assessed by the combined testing of odor identification, odor discrimination and olfactory threshold. Chem Senses. 1997; 22: 39-52.

9. Boyle JA, Frasnelli J, Gerber J, Heinke M, Hummel T. Crossmodal integration of intranasal stimuli: a functional magnetic resonance imaging study. Neuroscience. 2007; 149: 223-231.

10. Ashburner J, Friston KJ. Spatial normalization using basis function. In: Human Brain Function 2nd ed., Academic Press: Amsterdam, 2003.

11. Penny WD, Holmes AP. Random effects analysis. Human Brain Function. 2nd ed.; Amsterdam, Academic Press: 2003.

12. Hummel T, Barz S, Lotsch J, et al. Loss of olfactory function 
leads to a decrease of trigeminal sensitivity. Chem Senses. 1996; 21: 75-79.

13. Walker JC, Kendal-Reed M, Hall SB, et al. Human responses to propionic acid. II. Quantification of breathing responses and their relationship to perception. Chem Senses. 2001; 26: 351-358.

14. Savic I, Gulyas B, Berglund H. Odorant differentiated pattern of cerebral activation: comparison of acetone and vanillin. Hum Brain Mapp. 2002; 17: 17-27.

15. Doty RL. Intranasal trigeminal detection of chemical vapors by humans. Physiol Behav. 1975; 14: 855-859.

16. Apkarian AV, Bushnell MC, Treede RD, Zubieta JK. Human brain mechanisms of pain perception and regulation in health and disease. Eur J Pain. 2005; 9: 463-484.

17. Bensafi M, Iannilli E, Gerber J, Hummel T. Neural coding of stimulus concentration in the human olfactory and intranasal trigeminal systems. Neuroscience. 2008; 154: 832-838.

18. Treede RD, Kenshalo DR, Gracely RH, Jones AK. The cortical representation of pain. Pain. 1999; 79: 105-111.

19. Devinsky O, Morrell MJ, Vogt BA. Contributions of anterior cingulate cortex to behaviour. Brain. 1995; 118 ( Pt 1): 279-306.

20. Squire LR, Stark CE, Clark RE. The medial temporal lobe. Annu Rev Neurosci. 2004; 27: 279-306.

21. Savic I, Berglund H. Passive perception of odors and semantic circuits. Hum Brain Mapp. 2004; 21: 271-278.

22. Mainland JD, Johnson BN, Khan R, Ivry RB, Sobel N. Olfactory impairments in patients with unilateral cerebellar lesions are selective to inputs from the contralesional nostril. J Neurosci. 2005; 25: 6362-6371.

23. Applegate LM, Louis ED. Essential tremor: mild olfactory dysfunction in a cerebellar disorder. Parkinsonism Relat Disord. 2005; 11: 399-402.

24. Connelly T, Farmer JM, Lynch DR, Doty RL. Olfactory dysfunction in degenerative ataxias. J Neurol Neurosurg Psychiatry. 2003;
74: $1435-1437$.

25. Frasnelli J, Schuster B, Hummel T. Interactions between olfaction and the trigeminal system: what can be learned from olfactory loss. Cereb Cortex. 2007; 17: 2268-2275.

26. Royet JP, Zald D, Versace R, et al. Emotional responses to pleasant and unpleasant olfactory, visual, and auditory stimuli: a positron emission tomography study. J Neurosci. 2000; 20: 7752-7759.

27. Rolls ET, Grabenhorst F, Parris BA. Neural Systems Underlying Decisions about Affective Odors. J Cogn Neurosci. 2010; 22: 10691082.

28. Savic I. Brain imaging studies of the functional organization of human olfaction. Neuroscientist. 2002; 8: 204-211.

29. Gottfried JA, Winston JS, Dolan RJ. Dissociable codes of odor quality and odorant structure in human piriform cortex. Neuron. 2006; 49: 467-479.

\section{Emilia Iannilli, $\mathrm{PhD}$}

Smell \& Taste Clinic

Department of Otorhinolaryngology

University of Dresden Medical School

Fetscherstrasse 74, 01307

Dresden

Germany

Tel: +49-351-458-4189

Fax: +49-351-458-4326

E-mail: Emilia.Iannilli@uniklinikum-dresden.de 